\title{
Research on the Timing Synchronization Technology of 4G Mobile Communication
}

\author{
Chen Wen-ting ${ }^{1, \text { a }}$ \\ ${ }^{1}$ Chongqing College Of Electronic Engineering, Chongqing 401331, China \\ a37464351@qq.com
}

Keywords: Timing Synchronization Technology, 4G Mobile Communication

\begin{abstract}
Fourth generation mobile communication technology (4G) have more features than the third-generation standards, the fourth generation of mobile communications technology to provide wireless services in different fixed, wireless internet and cross-nowhere band network, broadband can be anywhere Internet access can be provided in addition to positioning the timing information and communication outside the integrated functions of data acquisition, remote control, multi-function integrated broadband mobile communication system or multimedia mobile communication systems. This article will focus on 4G mobile communication technology and analysis timing synchronization techniques.
\end{abstract}

\section{Introduction}

Currently, the International Telecommunication Union (ITU) and major telecommunications operators are working on 4G mobile communications technology with high-speed transmission rate of the 4G mobile communication systems with MIMO network, Adhoc networks and wireless local area network by combining up to $100 \mathrm{Mbit} / \mathrm{s}$ of data transfer rate, and can achieve seamless connection between different types of mobile communications services and wireless networks and systems. Thus, 4G mobile communications technology has the following advantages: data transfer rates of up to $100 \mathrm{Mbit} / \mathrm{s}$; high spectral efficiency; with good privacy and compatibility, to ensure compatibility with other types of networks. With the rapid development of mobile communication technology, mobile communication services for the people demand more and more, including dynamic high-speed transmission services, seamless roaming services, but these needs based on $3 \mathrm{G}$ mobile communication technology background also failed to achieve. Thus, communications experts and scholars will study sites are all located on 4G mobile communication systems in the hope that it is difficult to achieve all-round service provided, so that different types of communication networks can be seamless, compatible with each other.

Timing synchronization system unit is in the process of building a network constantly raised, and therefore have their basic needs may be limited to $4 G$ network features, but the subject of consideration to maximize the versatility of the apparatus. System means basic needs can be divided into functional requirements, both indicators of demand. Specifically, the clock synchronization system clock means to be able to achieve long-distance transmission of the basic requirements, and the use of optical fiber transmission media medium. Since the base station installation location often subject to the aggregate wiring planning and environmental interference, so the engineering for all long-distance transmission using optical fiber medium. For example, in a wireless communication network is widely Construction RF optical zooming device and fiber optic repeater. Timing synchronization system means also need to meet the $4 \mathrm{G}$ system clock index requirements, if they cannot meet the requirements of the $4 \mathrm{G}$ system design device does not have the availability. In fact, the ultimate goal of this design is to meet the sub-microsecond level clock synchronization accuracy.

Timing synchronization system means from a practical point of view needs to realize is divided into two parts, one installed outdoors, integrated GPS receiver or receivers Compass and clock synchronization signal transmission module, the paper said that this part of the device as the clock outdoor unit (OCU). Another part installed indoors, inside a integrated clock recovery module and a 
clock distribution module, wherein a clock distribution module is optional, this paper called the device clock indoor unit (ICU). In order to meet the current needs of network construction, the current clock outdoor unit to approximate the appearance of a closed cavity GSP antenna and an indoor unit to be compatible with the base station apparatus, the subject of relying on an optional hot-swappable cards as base hardware platform.

\section{The Overview of 4G Mobile Technology}

4G mobile communication technology had been proposed in the last century, the International Telecommunication Union will also "IMT-2000" and beyond the system as a work plan, and proposed to complete the initial commercial 4G in 2010 in the plan. Entering the new century, with advances in network communication technology and the rapid development of computer technology, IMT-2000 system has become the focus of the study, with the support of the International Telecommunication Union in 2000 in Canada, set up a special research group, in order to the working group will examine the worldwide harmonization. 4G technologies are increasingly concerned about our country and the corresponding research work has been officially included in the national 863 Project.

It can be understood from the following fourth-generation mobile communication systems: (1) is a new wireless communication system, the system is built on a new frequency band; (2) to the packet data based on information to achieve its high transfer rate; (3) a true "global Unity"; (4) based on the new constitution of the network system, or that it would be part of the new wireless network wireless access; (5) will not be a simple communication system, It is the integration of digital communications, digital audio / video system to accept the new network and counted.

Fourth generation mobile communication has its innovations, and previous generations of the systems are very different, the system network architecture to technology-based routing, in the conventional system, the core network is only one, namely the role of the mobile network. And in the fourth generation communication system, it is more like a unified fixed network, a mobile management functions, and can be wired, wireless connection phase. When connecting with a wireless access point with a variety of options, such as wireless local area networks, cellular base stations and other systems, these access methods, although slightly different, but the structure is the same signaling, information about the format, usually with IP packets and two kinds of ATM cells. In addition, wireless access points, there are many changes, the user can access at any time, but also in the communication process between the access points to complete the conversion. It should be noted that the core network is significant and we need to master the specific location of the user in real time, to identify the user's identity.

\section{G Mobile Communication Techniques}

Currently, the third generation mobile communication has begun large-scale commercial, but the technical limitations of its own has already attracted attention, and therefore the world's communications industry experts are already eyeing the post-3G technology that is $4 \mathrm{G}$ technology. Generating 3G to 4G technology evolution and the development of a series of new mobile communications technologies, including OFDM technologies, smart antennas, MUD technology.

OFDM Technology. As a special technique, OFDM is a multicarrier transmission and reception of the work to achieve the signal, the principle of this technique is that, at a certain frequency domain, the system will set a good channel to be divided to form a plurality of orthogonal sub channel, narrowband modulated transmission job or in the sub-channel is completed, the channel width is generally slightly faster than the band width of the signal number. By narrowband modulated, you can reduce the speed of high-speed serial data, making the low-speed sub-stream, by means of which the sub data subcarrier streams modulate these converted, so that they are mutually orthogonal, and ultimately parallel transmission. OFMD technology since it is possible interference, spectrum efficiency and attracted widespread attention, has become one of the key technologies of the future mobile communication systems. 
Smart Antenna Technology. Smart antenna by weighting the amplitude and phase of the antenna array element signal to change the pattern shape of the array, having lateral and zeroing function, it is possible the antenna array pattern alignment of the main lobe of the user signal direction of arrival, and adaptive real-time tracking signal, while the direction of arrival or interference the alignment signal, thereby suppressing the interfering signal to improve the signal to noise ratio, improve the performance of the entire communication system, and to identify the direct wave and reflected waves in different incident direction.

MUD Technology. The technology is a multi-user detection technology, when more users will inevitably occupy a certain channel, while the respective signal amplitude and other factors vary, MUD technology combined with time, phase, and signal strength information and other factors will be some users consider, on this basis, a single signal condition is detected the user to achieve optimal joint detection between users.

Wireless ATM Technology. WATM basic concept is the use of a standard ATM cell for network-level functionality, while adding a wireless radio link header / trailer protocol for the dedicated radio channel sub-layer (medium access control, data link control and wireless network control).

Ipv6 Technology. IPv6 address length will increase four-fold, from IPv4's 32 bits to 128 bits. IPv6 not only solves the problem of IP address is not enough, but also improve the safety and quality of service of the network. The main of these features: expanded IP address space; enhanced authentication and privacy; simplified header format, to strengthen support for the extension header and options section; the data stream identifier; improved mobile network performance and real-time communications.

\section{Timing Synchronization System Communication Model Design}

In the field of communications, information exchange occurs between different types of entities. The entity is anything capable of sending and receiving information. However, the two entities cannot simply send bit streams to each other and want them to understand the bit stream. To communicate between at least two entities must agree to the use of a protocol. This protocol is a set of rules that control data communications. Protocol defines what you want to transfer, how to communicate and when to communicate. Three key elements of the agreement is the syntax, semantics, and synchronization. Grammatical structure is the order of significance live format of the data, and the data appears. Semantics is the meaning of each part of the bit stream. A special bit pattern should explain how, based on this should be interpreted and how to deal with. Synchronization has two characteristics: when data should be sent and the data should be transmitted much faster. International Organization for Standardization work S0 by the multinational groups to form for the worldwide agreement, proposed ISO standard can cover all aspects of the network, namely the OSI model. The OSI model is a hierarchical framework network system design, so that the type of communication system. It includes seven separate but related layers, in which each layer defines the process of transmitting information over a network. But because of its emphasis reflects the versatility of various networks, so far there is no will to implement a network of all seven layers of the OSI functionality. Similarly, the timing synchronous communication system is a complete system-level design, this communication system also work in strict accordance with the OSI seven-layer communication functions S0 plan. With the subject in depth, this paper proposes a transfer for remote synchronous clock signal of standardized information exchange, in this paper called the clock to pull away the technical specifications. Pull away the clock technical specifications into physical layer, a media access control layer and application layer. The physical layer is divided into PMD, PMA, PCS sub-layer, standardized physical characteristics and physical layer encoding and decoding.

PMN Chinese name for the physical media dependent sub-layer, which standardizes the physical network media-related characteristic index. PMD sub-layer functionality is supported between the PMA sub-layer and media exchange serialized bit character codes.

PMA Chinese name for the physical media attachment sub-layer, PMA sub-layer provides serial 
Service Interface PCS and PMD layer. It is connected to the PCS sub-layer called PMA service interface. Further PMA sub-layer also isolated from a received bit stream data received for the correct alignment of the symbol (delimitation) symbol timing clock., These electrical signals into a form suitable for transmission over a particular medium. PMD is the lowest sub-layer of the physical layer standard provides physical layer is responsible for transmitting and receiving signals from the media.

PCS Chinese name for the physical coding sub-layer. PCS sub-layer located Reconciliation sub-layer (between (PMA) sub-layer. PCS after completion of the well-defined Ethernet MAC function mapping by GM workers work and physical media access layer to the existing coding and physical layer signal system up function.

\section{Timing Synchronization System Unit Hardware and Software Design}

The clock synchronization system is divided into an outdoor unit and an indoor unit clock synchronization, clock synchronization has been determined the outdoor unit commissioned the Association of manufacturers to develop, so in this paper will no longer be described. It is seen by the indicators of demand, clock synchronization system to meet the order of sub-microsecond clock accuracy, so the system transmission rate to reach hundreds of megabytes or more in order to meet the rate of the recovered clock accuracy. Since the system was first proposed and custom applications, there is no integrated chip can be directly used as the hardware platform, so this topic programmable logic devices to implement the physical layer functions. This logic devices use xilinx's FPGA chips. This series chip fully meets the needs of the system clock, and low cost are conducive to system clock synchronization means large-scale promotion.

Clock indoor unit and outdoor unit as a clock operable to maintain the equipment, you need software to manage and monitor status. First, we need to realize the clock to pull away message application layer protocol specified for resolution; secondly, the need to provide the operator interface to remote equipment monitoring and status reporting interfaces; and finally, the need to achieve the desired reference frequency tracking station for timing synchronization signal functions. Software-based company produced free scale embedded communications processor platform for development, real-time operating system on top of the processor core and peripherals management, of course, also supports multi-tasking or multi-threaded operation. This gives the upper software provides a wealth of input and output interfaces.

\section{Conclusions}

After decades of development of wireless mobile communication, it has a profound impact and change people's production and life. Future wireless mobile communication system will be able to achieve it at any time, any place and any person in any way desire to communicate. To achieve this goal, a variety of new communications technologies are emerging. How to use the limited spectrum resources to provide reliable high-speed broadband data traffic is concerned for the current academia and industry.

\section{References}

[1] Huifang Zhou: Information and Communication, Vol. 6 (2011) No 53, p.25-26

[2] Hongli Zhang: Information Technology, Vol. 12 (2013) No 27, p.74-76

[3] Qin Guo: Computer and Network, Vol. 1 (2011) No 33, p.11-14

[4] Jieming Liu: Guangxi Normal University, Vol. 3 (2010) No33, p.121-124

[4] Zhang Minru: Science Technology and Engineering, Vol. 13 (2014) No10, p.52-56 